
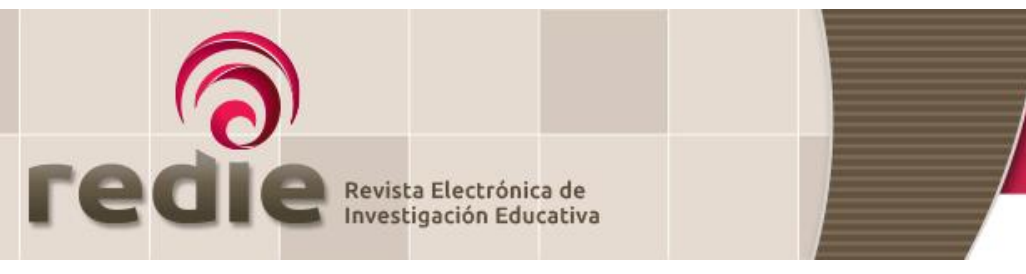

Vol. 20, Núm. 4, 2018

\title{
Percepción de los estudiantes de educación inicial frente al desarrollo de experiencias formativas en modalidad A+s
}

\author{
Perception of Early Education Students with Regard \\ to the Development of Formative Experiences \\ Based on the Learning and Service Modality
}

Pilar Andrea Uribe Sepúlveda (*) pilar.uribe@uautonoma.cl

(*) Universidad Autónoma de Chile

(Recibido: 15 de febrero de 2017; Aceptado para su publicación: 3 de abril de 2017)

Cómo citar: Uribe. P. A. (2018). Percepción de los estudiantes de educación inicial frente al desarrollo de experiencias formativas en modalidad A+S. Revista Electrónica de Investigación Educativa, 20(4), 110-122. https://doi.ora/10.24320/redie.2018.20.4.1826

\begin{abstract}
Resumen
En este trabajo se analizan las percepciones de estudiantes en formación inicial docente de educación infantil sobre el aporte de incluir la modalidad de Aprendizaje y Servicio (A+S) en asignaturas de práctica pedagógica en contextos interculturales rurales. Los resultados presentan percepciones positivas en el desarrollo personal y profesional de las estudiantes, destacando el fortalecimiento de competencias genéricas asociadas a la ética, valoración a la diversidad y trabajo en equipo, la dimensión moral, valórica y actitudinal que éstas pueden; así como un vuelco en las representaciones sociales asociadas al trabajo con adultos y familia.
\end{abstract}

Palabras clave: Formación de profesores, aprendizaje y servicio, educación intercultural.

\section{Abstract}

This study analyzes the perceptions of students of initial teacher training in childhood education regarding the contribution provided by the inclusion of the Learning and Service $(L+S)$ modality in subjects involving teaching practice in intercultural rural contexts. The results show positive perceptions in students' personal and professional development, with particular emphasis on strengthened generic ethical competence; an appreciation of diversity and teamwork; the moral, value-based and attitudinal dimension experienced by students; and a shift in social representations associated with working with adults and families.

Keywords: Teacher training, learning and service, intercultural education.

\section{Introducción}

La evolución y características de la formación inicial docente durante los últimos 15 años en Chile ha sido objeto de diversos estudios que se centran en el análisis de las políticas de formación docente (Cox, Meckes y Bascopé, 2010), efectividad para producir conocimiento pedagógico general y de contenido 
(Ávalos y Matus, 2010), la relación entre calidad de las instituciones en que se albergan los programas de formación docente y el nivel de conocimiento de los futuros profesores (Pedraja et al., 2012), hasta políticas destinadas a "atraer, desarrollar y retener profesores efectivos" (OCDE, 2005; Barber y Mourshed, 2007). Las instituciones formadoras desde el año 2004 a la fecha han vivido distintos procesos de fortalecimiento en sus programas, los que van desde el financiamiento desde el Ministerio de Educación (MINEDUC) a mejorar los procesos de formación docente en el marco del programa de Mejoramiento de la Calidad de la Educación Superior (MECESUP), la promulgación de la Ley de Aseguramiento de la Calidad de la Educación Superior en el año 2006, el monitoreo de conocimientos disciplinarios y profesionales de los futuros profesores a través de la aplicación de la Prueba Inicia el año 2008; hasta la postulación de Convenios de Desempeño para las instituciones formadoras de profesores desde el año 2012. No obstante los esfuerzos desarrollados por el Estado chileno, la percepción de quienes siguen de cerca los procesos de formación docente chilenos ha alertado respecto a una serie de condiciones problemáticas. Entre estas condiciones destaca que los programas de formación han crecido más en número que en la calidad de las instituciones formadoras, lo que afectaría la competencia de sus egresados y, por lo tanto, la formación del "ser persona" y cómo el profesional de la educación se involucra y hace cargo de las necesidades reales de su comunidad (Ávalos, 2014).

La sociedad actual demanda a las universidades formar profesionales competentes que sirvan responsable e íntegramente a las personas para que éstas contribuyan a un mayor desarrollo de la sociedad, sociedad que comparada con las anteriores es cualitativamente más distinta, compleja e incierta (Sagastizabal, 2009). En ese contexto de incertidumbre, el concepto de profesional competente apunta a definir el manejo de habilidades, destrezas, procesos y estrategias que involucran todas las dimensiones de la persona, vinculadas a su profesión en un contexto social igualmente complejo y pluridimensional. La modalidad de Aprendizaje y Servicio (A+S) aporta a este mandato social, sobre todo si se entiende éste como una filosofía que busca que los contenidos curriculares se potencien, se aprendan y se apliquen en contextos solidarios y de servicio a la comunidad (Tapia, 2006).

El aspecto clave para considerar a una experiencia educacional como A+S, y no sólo como un voluntariado, es el equilibrio que debe darse entre los aprendizajes de los estudiantes con el servicio orientado a una necesidad real de una comunidad, basado en una metodología experiencial con un enfoque más participativo y democrático, donde las actividades de servicio se integran con el currículo de formación mejorando la calidad de ambos. Esta metodología no implica agregar más elementos a los planes de estudio, sino más bien buscar formas creativas de unir los contenidos con las necesidades de una comunidad (Eyler, 2009; Furco y Billing, 2002; Jouannet, Salas y Contreras, 2013; Tapia, 2006). Las iniciativas universitarias que se enfocan en entregar un servicio solidario (como voluntariados y campañas) no podrían ser consideradas A+S, ya que no tienen por foco el aprendizaje de los estudiantes, sino el servicio solidario ofrecido.

Los aportes y beneficios que obtiene un estudiante universitario que vive la experiencia de formación de aprendizaje y servicio, son variadas y apuntan a aspectos conceptuales, procedimentales y actitudinales (Delors, 1994).

En relación con el aspecto conceptual, Jenkins y Sheehey (2011) plantean que esta metodología aumenta la adquisición de conocimientos disciplinares en los estudiantes, a la vez que fomenta la confianza y el compromiso social en los estudiantes; en cuanto al aspecto procedimental hay estudios que indican que esta metodología propicia el desarrollo de habilidades de liderazgo y valores como la justicia social (Groh, Stallwood y Daniels, 2011). Finalmente y asociado con el aspecto actitudinal, Cooper, Cripps y Reisman (2013) señalan que esta experiencia de formación impacta positivamente en la percepción de su rol como un agente de cambio, asociando a esto el compromiso cívico y las actitudes altruistas.

En resumen, el impacto en el desarrollo integral de la persona no se puede desconocer, y a la vez aporta al desafío de la educación que señalan que ésta tiene la necesidad de transmitir, masiva y eficazmente, volúmenes cada vez mayores de conocimientos teóricos y técnicos, adaptados a la civilización cognoscitiva, porque son las bases de las competencias del futuro (Delors, 1994). 


\subsection{Aprendizaje y servicio en contextos educativos interculturales}

Como se señala desde el Proyecto de Intervención Comunitaria Intercultural (2013), el verdadero éxito de la educación consiste en formar ciudadanos capaces de mejorar la sociedad, no sólo su currículum personal. Dicha demanda, Sagastizabal (2009) la asume como un desafío desde la sociedad, pero la autora es clara al señalar que es en los espacios educativos donde esto se debe propiciar, en este aspecto las instituciones educativas tienen la responsabilidad de observar con fineza las demandas que hoy la sociedad tiene en cuanto a diversidad, interculturalidad y humanidad, entre otros, sólo de esta forma las instituciones educativas se convierten en verdaderos espacios de formación ciudadana.

Independientemente del enfoque empleado en las prácticas de educación intercultural, ya sea en temáticas étnicas o aquellas asociadas a fenómenos migratorios, en ésta se promueve el aprendizaje efectivo de competencias y conocimientos que faciliten el diálogo y la convivencia, fomentado habilidades concretas que permitan el reconocimiento, valorización y entendimiento de la diversidad cultural en sus múltiples formas y contextos (Mason, 2015).

En este tipo de aprendizaje el desarrollo de una sensibilidad intercultural y la adquisición de competencias interculturales específicas que favorezcan el diálogo toman cada vez mayor protagonismo (Bennett, 1986; 1993; 2004); bajo esta perspectiva, la modalidad de aprendizaje y servicio unido a la educación intercultural es el escenario perfecto para el desarrollo de este aprendizaje, pues la educación intercultural es reconocida como "una forma de interacción equitativa entre diversas culturas y la posibilidad de generar expresiones culturales compartidas, adquiridas por el diálogo y el respeto mutuo" (UNESCO, 2006, p. 17), donde el desarrollo del diálogo depende de cómo se lleven a cabo ciertas condiciones e interacciones específicas desde criterios que implican conocer, involucrarse y -una vez que hay un acercamiento- propiciar relaciones simétricas en términos de conexiones de poder que permitan mantener la dualidad en el seno de la unidad (Molina, 2002; Sorrells y Nakagawa, 2008; UNESCO, 2006).

El Centro Latinoamericano de Aprendizaje y Servicio Solidario [CLAYSS] (2014) y Tapia (2006) sostienen que un proyecto de intervención con la comunidad se desarrolla en tres etapas: a) acercamiento, reconocimiento y diagnóstico de la realidad; b) ejecución de la propuesta; y c) cierre y evaluación. Todo esto acompañado de procesos reflexivos, comunicación entre los involucrados y una mirada evaluativa. Dicha propuesta es coherente con la oferta formativa de los procesos de práctica pedagógica en la cual se desarrolla esta investigación, pues las asignaturas que constituyen este proceso se definen como espacios que favorecen el encuentro del estudiante con la identidad personal y construcción de la identidad profesional, para ello la práctica pedagógica se desarrolla bajo una concepción en espiral del aprendizaje (Schön) y se orienta a la adquisición de competencias que le permitan al estudiante la asunción progresiva de la profesión docente como una actividad de investigación permanente, especialmente en el contexto de la gestión de planes de desarrollo educativos generados desde las necesidades de los contextos socioeducativos, donde la reflexión e indagación del desempeño se llevará a cabo a través de experiencias de investigación-acción tomando como base el modelo reflexivo de Korthagen.

\section{Método}

La Universidad Católica de Temuco, desde el año 2004, incorpora en su currículo la línea de Formación Humanista Cristiana (FHC) con actividades de libre elección, dicha línea curricular es un soporte institucional básico que busca ayudar a que cada carrera integre en las actividades docentes la vocación de servicio a la sociedad, la valoración y respeto hacia la diversidad, y un sólido sentido de actuación ética; actividades que desde el 2009 se reemplazaron por "Actividades de servicio comunitario".

En el 2015 la carrera de Educación Parvularia participó en una actividad piloto de A+S modalidad trayectoria y servicio, que se incorpora en el cuarto año de formación (6o.-7o. semestre), específicamente en asignaturas vinculadas a la mención de educación intercultural con el fin de incorporar al debate del campo de la investigación indagaciones vinculadas con el diseño de propuestas formativas de impacto social, su posterior implementación, evaluación y problematización de fenómenos educativos. 
Hasta antes de la implementación de la modalidad de A+S en el currículo de formación de las estudiantes de Educación de párvulos de la UCTemuco, se evidencia que los razonamientos que logran las estudiantes en sus prácticas pedagógicas se asocian mayoritariamente a niveles de desarrollo reflexivo del propio desempeño. Es por ello que la incorporación de la modalidad A+s en el currículo permite comenzar un seguimiento sobre categorías como sensibilidad cultural y competencias interculturales de un grupo de estudiantes para llegar a conocer el grado de apropiación (percepción y razonamiento) que los profesionales en formación construyen desde sus prácticas progresivas en la vinculación con el aprendizaje y servicio, a partir de preguntas problematizadoras que se derivan de las permanentes evaluaciones en el currículo y que apuntan a identificar qué perfil tiene un profesional competente en condiciones sociales e históricas en ocasiones adversas y diferentes a otros contextos. Este problematiza las condiciones que limitan los tipos de perfiles competentes, sobre todo en contextos de interculturalidad. La tabla I muestra el itinerario formativo que acompaña al desarrollo de las actividades de $A+S$.

Tabla I. Itinerario formativo de actividades A+S

\begin{tabular}{|c|c|c|c|}
\hline \multicolumn{2}{|c|}{$\begin{array}{l}\text { Mención " Interculturalidad" } \\
\text { 40. año / 6o. semestre }\end{array}$} & \multicolumn{2}{|c|}{$\begin{array}{c}\text { Mención "Interculturalidad" } \\
\text { 40. año / 7o. semestre }\end{array}$} \\
\hline $\begin{array}{l}\text { Valores y saberes } \\
\text { en educación } \\
\text { intercultural }\end{array}$ & $\begin{array}{l}\text { Programas } \\
\text { y experiencias } \\
\text { de educación } \\
\text { intercultural }\end{array}$ & $\begin{array}{l}\text { Desarrollo } \\
\text { de competencias } \\
\text { interculturales }\end{array}$ & $\begin{array}{l}\text { El diálogo como } \\
\text { encuentro con el } \\
\text { otro }\end{array}$ \\
\hline \multicolumn{2}{|c|}{$\begin{array}{l}\text { Taller Pedagógico V sec } 02 \\
\text { Curso desarrollo experiencia A+s }\end{array}$} & \multicolumn{2}{|c|}{$\begin{array}{c}\text { Taller Pedagógico VI sec } 02 \\
\text { Curso desarrollo experiencia A+s }\end{array}$} \\
\hline
\end{tabular}

Esta experiencia de trabajo de A+S se desarrolló durante el 10. y 2o. semestre académico del 2015, con la participación de 26 mujeres de tercer año de la carrera de Educación Parvularia de la mención de educación intercultural de la Universidad Católica de Temuco, distribuidas en 13 parejas de prácticas pedagógicas progresivas y un docente supervisor; su fin fue conocer el grado de percepciones y razonamientos de las estudiantes de la muestra que han sido formadas con ciertas características y el seguimiento de los mismos en determinados contextos educativos donde desarrollan sus prácticas.

Los escenarios de práctica fueron escuelas rurales municipales en contexto indígena de la comuna de Freire (Chile). A continuación se presenta el listado de escuelas y comunidades que participaron durante el período, así como la cantidad de parejas de práctica que asistió a cada uno de ellos:

Tabla II. Distribución de centro de prácticas

\begin{tabular}{l|c}
\hline Centro de práctica & $\begin{array}{l}\text { Cantidad de parejas } \\
\text { de prácticas }\end{array}$ \\
\hline Truf Truf & 2 \\
Ñirrimapu & 2 \\
Tromen Quepe & 1 \\
Hermano Pascual & 1 \\
Fundo Maquehue & 1 \\
Licanco & 2 \\
Escuela Metrenco & 2 \\
Wekintün & 2 \\
\hline
\end{tabular}

Los instrumentos de recolección de datos fueron un grupo focal, al momento de cierre del primer semestre; y una encuesta de percepción que se aplicó al cierre del 2o. semestre. Ambos instrumentos se elaboraron en función a 3 categorías que apuntaron a los objetivos del trabajo, y que a la vez fueron parte del proceso de trabajo vivenciado por las estudiantes: 1) Principales competencias genéricas desarrolladas a lo largo de la experiencia A+S; 2) Aprendizajes construidos del trabajo comunitario; y 3) Aportes de la experiencia A+S a la formación profesional. 
El procedimiento de análisis se basa en el análisis de contenido, éste centra su interés en la lectura e interpretación del contenido manifiesto y latente presente en los testimonios de los participantes (Ruiz, 1996). Esta técnica de análisis entregó la posibilidad de realizar un procesamiento metódico de los datos, con el propósito de generar inferencias que fueron reproducibles y válidas (Krippendorf, 1990; Quivy y Campenhoudt, 1998).

La identificación de las categorías de contenido se realizó por medio de: a) La codificación abierta que permitió una apertura a la indagación en los datos, para así generar un conjunto emergente de categorías; b) El método comparativo constante enfocado a la búsqueda de semejanzas y diferencias presentes en los datos; c) La saturación teórica, proceso relacionado con la búsqueda suficiente de información que favorezca la interrogación de las categorías que emerjan en el análisis; d) La triangulación de datos para confrontar los resultados del proceso de investigación; y e) La codificación axial para reducir el conjunto inicial de categorías y propiciar un análisis intensivo a la base de la categoría central de la investigación (Trinidad, Carrero y Soriano, 2006).

Para complementar el análisis se utilizaron elementos de la Teoría Fundamentada, la codificación abierta y axial, para tratar y expresar la información obtenida en forma de conceptos, luego se redujo el conjunto inicial de categorías siguiendo los lineamientos del método comparativo constante, la saturación teórica y la triangulación de los contenidos (Flick, 2004; Glaser y Strauss, 1967; Strauss y Corbin, 2002; Trinidad, Carrero y Soriano, 2006). Este proceso se apoyó con el uso del software de análisis cualitativo Atlas.ti 5.0, que permitió organizar y tratar con mayor fluidez los datos de la investigación. De esta manera, el análisis de contenido siguió un proceso riguroso de lectura científica de los datos que permitió la identificación de unidades de registro (códigos), que fueron sistematizadas y clasificadas en categorías de contenido.

\section{Resultados}

Las respuestas obtenidas en los instrumentos y procedimientos utilizados durante el trabajo permitieron recoger con detalle las percepciones y razonamientos de la experiencia de A+S en las estudiantes a partir de categorías como sensibilidad cultural y competencias interculturales, que permiten al profesional no sólo orientar su labor hacia el aprendizaje sino a reconocer aspectos como: el aprendizaje esperado, la práctica social y la competencia por lograr. Los principales resultados de acuerdo con las categorías propuestas fueron: competencias genéricas desarrolladas a lo largo de la experiencia A+S, aprendizajes construidos del trabajo comunitario y aportes de la experiencia A+S a la formación profesional.

\subsection{Principales competencias genéricas desarrolladas a lo largo de la experiencia A+S}

De acuerdo a las respuestas obtenidas se puede señalar que, de las competencias trabajadas en esta experiencia de A+S, las estudiantes señalan que: 1) actuación ética, 2) valoración y respeto a la diversidad y 3) trabajo en equipo, son aquellas con las que pudieron vivenciar y lograr con mayor impacto. A continuación se define cada una de las competencias valoradas por las estudiantes, y los indicadores con los cuales las estudiantes fundamentaron esta selección:

1) Actuación ética: Demuestra sentido ético sustentado en principios y valores de justicia, bien común y de la dignidad absoluta de la persona humana, que le instan a servir a la sociedad responsablemente en respuesta a las necesidades que ella le demanda como persona, ciudadano y profesional. Esto implica, por una parte, poner atención a la dimensión subjetiva del discernimiento ético como capacidad intelectual y volitiva de valoración de la realidad con base en principios éticos universales. Por otra parte, considera la dimensión objetiva de los valores, privilegiando la justicia, el bien común y la dignidad humana.

Los indicadores con los cuales se evalúa el logro de esta competencia suponen:

- Habilidad para conocer y evaluar críticamente la realidad que y sus procesos sociales, especialmente desde la perspectiva de la propia disciplina y profesión;

- Capacidad de tomar decisiones que permitan actuar personal y colectivamente en consonancia 
con el análisis moral realizado;

- Predisposición positiva, en cuanto persona, ciudadano y profesional, hacia la actuación socialmente responsable.

- Por último, el desarrollo de la competencia de actuación ética en una institución como la nuestra, que se entiende a sí misma desde la perspectiva de la responsabilidad social universitaria, implica el reforzamiento de la identidad personal y profesional de sus estudiantes a partir del servicio a la sociedad, en especial a los más pobres, y la colaboración en la resolución de los grandes problemas del desarrollo humano.

2) Valoración y respeto hacia la diversidad: Reconoce al otro en su dimensión humana, comprendiendo que las diferencias sociales, culturales y de capacidades enriquecen la convivencia sin incurrir en prácticas discriminatorias. Implica el comprender y aceptar la diversidad social, cultural, religiosa, de género y de capacidades como un componente enriquecedor personal y colectivo. Considerando la vocación regional de nuestra identidad institucional, tiene particular relevancia descubrir que las diferencias culturales y étnicas que nos caracterizan como región son complementarias. Busca dialogar creativamente con su entorno cultural y, a la vez, promover y facilitar el diálogo interétnico e intercultural. Por otra parte, la valoración y respeto hacia la diversidad permite también convivir sin incurrir en discriminaciones.

Los indicadores con los cuales se evalúa el logro de esta competencia suponen:

- Habilidad de análisis y perspectiva social.

- Comprender la forma de ver la realidad que tiene el otro.

- Habilidad de convivencia social sustentada en relaciones interpersonales integradoras.

- Disposición positiva y reconocimiento del otro como legítimo otro.

3) Trabajo en equipo: Demuestra integración y colaboración de forma activa en la consecución de objetivos comunes con otras personas, áreas y organizaciones. el trabajo en equipo implica que los individuos desarrollan actitudes de simpatía y empatía, comunión profunda con los intereses y procesos de su equipo, generando un vínculo afectivo. Al mismo tiempo, en la medida que los individuos interactúan, participan y hacen suyos los propósitos y objetivos de su equipo, entonces el vínculo con su grupo de pertenencia es también efectivo. Busca identificar los comportamientos asociados a los equipos de trabajo efectivos, asumiendo el hecho de que existen actividades específicas de los grupos que suponen avances a lo largo del tiempo, como son la toma de decisiones, los procesos de comunicación, la resolución de conflictos, etc. En este sentido, un individuo competente en trabajo en equipo es quien potencia las estructuras comunicativas y organizativas del grupo para hacerlo más eficaz en función de los objetivos de tarea propuestos y los fines comunes para los que se constituyó el equipo.

Los indicadores con los cuales se evalúa el logro de esta competencia suponen: a) habilidad para identificar objetivos y tareas comunes de un grupo de trabajo y reconocer las competencias distintivas en los distintos integrantes; b) habilidades sociales: comunicación explícita y cordial, adecuación al contexto, escucha activa, motivación; y c) actitud colaborativa y adscripción a los valores declarados por el equipo, como normativa cultural para las relaciones humanas.

\subsection{Aprendizajes construidos del trabajo comunitario}

Los aprendizajes que las estudiantes destacan de esta experiencia de formación, se asocian a contenidos y estándares que hoy el currículo chileno solicita a una educadora de párvulos; dentro de los testimonios que las estudiantes entregan destaca que los principales aprendizajes se enfocan en: 1) el diseño de la enseñanza, y cómo éste propicia conocer la cultura a mayor cabalidad; 2) desarrollo de la participación y liderazgo en pro del logro de aprendizajes significativos; 3) resolución de conflictos; y 4) capacidad de 
reflexión permanente de su práctica pedagógica (MINEDUC, 2012). A continuación se presenta la descripción de los aprendizajes, y el indicador a través del cual se demuestra el logro del mismo.

1) Diseño de la enseñanza. Este aprendizaje corresponde al estándar pedagógico 4, en el cual se indica que el educador de párvulos debe aprender a diseñar e implementar experiencias pedagógicas adecuadas para los objetivos de aprendizaje y de acuerdo al contexto. La educadora de párvulos que ha finalizado su formación inicial es capaz de planificar la enseñanza teniendo como foco el logro de objetivos de aprendizaje relevantes para los niños de manera coherente con el currículo nacional. Considera en su planificación las necesidades, intereses, conocimientos previos, habilidades, ritmos de aprendizaje, experiencias de los párvulos y el contexto en que se desarrollará la enseñanza, incluyendo los resultados de evaluaciones previas. Es capaz de planificar experiencias de aprendizaje y secuencias de actividades, dando a las niñas y niños el tiempo, el espacio y los recursos necesarios para aprender. Conoce las estrategias didácticas propias de cada ámbito del aprendizaje y es capaz de transformar este conocimiento en enseñanza (MINEDUC, 2012).

La educadora de párvulos en formación inicial demuestra el logro de este aprendizaje cuando: diseña planificaciones de distinto alcance temporal para lograr los aprendizajes esperados de acuerdo al currículo en los distintos ámbitos del aprendizaje.

2) Desarrollo de la participación y liderazgo en pro del logro de aprendizajes significativos. Este aprendizaje se vincula con el estándar 13 que habla de mantener relaciones profesionales colaborativas con distintos equipos de trabajo. La educadora de párvulos que ha finalizado su formación inicial comprende que su quehacer cotidiano implica la participación y liderazgo pedagógico en distintos equipos de trabajo, los cuales incluyen personal técnico, pares profesionales y otros profesionales. Asumiendo que las mejores oportunidades de aprendizaje y desarrollo de las niñas y los niños a su cargo dependen en gran medida de la conformación activa de un trabajo en equipo, propicia que éstos logren un funcionamiento armónico y sinérgico. Para ello, se involucra asumiendo el rol de líder pedagógico en cada uno, con el propósito de favorecer la adopción de decisiones apropiadas para la primera infancia y contribuir a la calidad de la Educación Parvularia del programa educativo donde se desempeña (MINEDUC, 2012).

La educadora de párvulos en formación inicial demuestra el logro de este aprendizaje cuando: a) participa en instancias de análisis y de intercambio con sus pares profesionales en torno a las decisiones pedagógicas a adoptar respecto del aprendizaje de los estudiantes a su cargo; y b) participa y colabora en equipos interdisciplinarios, asumiendo una perspectiva pedagógica fundamentada para mejorar las oportunidades de aprendizaje que se ofrecen las niñas y los niños a su cargo.

3) Resolución de conflictos. Este aprendizaje se asocia con el estándar 12, señalado por el MINEDUC (2012), que alude a la capacidad que debe lograr el estudiante para construir relaciones de alianza con la familia y la comunidad. La educadora de párvulos que ha finalizado su formación inicial comprende que las familias son las primeras educadoras y, por tanto, tiene claro que las mejores oportunidades de aprendizaje y desarrollo de los niños a su cargo no pueden prescindir de la participación de las familias en el proceso educativo. De este modo, atendiendo y valorando las características específicas de las familias de su comunidad, genera relaciones profesionales de respeto y valoración recíproca, involucrándolas en el proceso educativo del cual es responsable (MINEDUC, 2012).

La educadora de párvulos en formación inicial demuestra el logro de este aprendizaje cuando: a) fomenta relaciones de confianza y respeto mutuo con las familias de cada niña y niño a su cargo; b) integra diversos saberes culturales propios de las familias en experiencias de aprendizaje, reconociéndolos como miembros de la comunidad educativa y como primeros educadores de los niños; c) organiza y ofrece oportunidades de participación a las familias en experiencias de aprendizaje en el centro educativo y su continuidad en el hogar; y d) establece relaciones 
colaborativas con organizaciones y servicios de la comunidad circundante y más amplia, para enriquecer las oportunidades de aprendizaje de las niñas y niños.

4) Capacidad de reflexión permanente de su práctica pedagógica. Este aprendizaje está inserto en el estándar 9 que indica que el estudiante de educación parvularia debe aprender en forma continua y reflexionar sobre su práctica y su inserción en el sistema educacional. La educadora de párvulos que ha finalizado su formación inicial comprende que su desempeño profesional requiere dedicación continua a su aprendizaje. Es capaz de analizar y reflexionar individual y colectivamente sobre su práctica pedagógica y sobre los resultados de aprendizaje de los niños bajo su responsabilidad. Puede proponer cambios a partir de juicios fundados sobre la base de los estándares profesionales, los resultados de aprendizaje de los estudiantes, la retroalimentación de otros educadores, y de las necesidades y expectativas del establecimiento educacional. Está preparado para resolver problemas pedagógicos y de gestión y, a la vez, comprometer a múltiples actores en el logro de aprendizajes de calidad de los estudiantes (apoderados, familias y otros agentes) (MINEDUC, 2012).

La educadora de párvulos en formación inicial demuestra el logro de este estándar cuando: analiza críticamente su práctica pedagógica y la de otros educadores, en función de su impacto en el aprendizaje de los niños, y propone y fundamenta cambios para mejorarla.

\subsection{Aportes de la experiencia A+S a la formación profesional}

Los datos recogidos en esta categoría indican que las estudiantes, luego de vivir una experiencia de A+S en su formación inicial, valoran aspectos asociados a:

1) Inserción de una práctica pedagógica contextualizada, esto se da a partir de la colaboración efectiva y compromiso de las estudiantes y socios comunitarios.

2) Aprender del otro: la mirada de los socios comunitarios y su participación activa permite una retroalimentación constante al trabajo de los estudiantes, así como a la propuesta pedagógica.

3) El liderazgo que implica el trabajo con los socios comunitarios, permite vivir experiencias nuevas que en otras prácticas no se han vivenciado y que impactan en el desarrollo profesional de los estudiantes.

4) Generación de redes de apoyo efectivo que se establecieron entre los socios comunitarios y centros de prácticas.

5) Se produce un vuelco en las representaciones sociales asociadas al trabajo con adultos y familia.

\section{Discusión}

De los resultados de este trabajo se desprenden similitudes con los realizados por Asociación Nacional de Facultades y Escuelas de Contaduría y Administración [ANFECA] (2013), CLAYSS (2014), Ministerio de Educación, Ciencia y Tecnología [MECYT] (2006), Puig (2009), Tapia (2009) y Universia (2010), entre otros. La discusión de este trabajo tiene que ver con el reconocimiento positivo por parte de las estudiantes frente a una experiencia basada en $A+S$, aunque existen debilidades y aspectos a mejorar, éstos se asocian a aspectos referidos a conectividad territorial y distribución de centros de prácticas, entre otros.

Si bien la mayoría de las universidades en los años noventa indicaban que su meta era alcanzar la excelencia académica, a esta premisa se agregó el concepto de servicio:

Para algunas Universidades el objeto de su existencia es la excelencia académica. Nosotros consideramos que la razón de nuestra existencia es el servicio a la gente, y la excelencia académica es su mejor instrumento (MECYT, 2006, p. 11). 
En relación con la primera categoría (Principales competencias genéricas desarrolladas a lo largo de la experiencia A+S), las respuestas de las estudiantes apuntan a lo que se indicó en el II encuentro de rectores Universia, donde se plantea que:

(...) el compromiso, cohesión e inclusión social, diversidad cultural, la promoción del desarrollo social, el progreso y el bienestar frente a los problemas de desigualdad, como la inequidad, pobreza, género y sustentabilidad, requiere de la elaboración y renovación del desarrollo de programas y acciones formativas que se encaminen hacia el logro del fomento de valores y competencias profesionales en los programas formativos y de investigación (Universia, 2010, primer eje 1.1).

Un fenómeno común en cualquier sociedad del conocimiento, independientemente de su etapa de desarrollo, es la velocidad con que se produce el fenómeno de obsolescencia, debido principalmente a los grandes avances que se tienen en el campo de la ciencia y la tecnología. Esto afecta cualquier actividad humana, pero en donde se manifiesta con mayor claridad es en el campo de la actividad económica y en la educación. En el ámbito educativo se requiere de personas que tengan un acervo de habilidades y competencias indispensables para un desempeño pertinente, creando nuevas necesidades y dando pie al desarrollo de nuevas especialidades que atiendan la demanda (ANFECA, 2013).

Para la segunda categoría (aprendizajes construidos del trabajo comunitario) se indica que las experiencias de aprendizaje-servicio permiten a los estudiantes aplicar sus saberes al servicio de las necesidades de su comunidad. Simultáneamente, esta acción solidaria en contextos reales les permite interpelar sus conocimientos al contacto con la realidad, desarrollar nuevas competencias profesionales y para la participación ciudadana; situaciones que se reflejan en las respuestas de las estudiantes, quienes reconocen una serie de aprendizajes que aún en situación de prácticas hubiesen sido difícil lograr, el desarrollo de éstos las estudiantes lo asocian mayoritariamente al aporte de la metodología de A+S, pues este tipo de experiencias permiten realizar una contribución concreta y evaluable a la vida de una comunidad, y también mejorar la calidad e inclusión de la propuesta educativa formal y no formal (CLAYSS, 2014); a continuación se presentan algunas textualidades que dan cuenta de lo aquí expuesto:

1) La explicación frente al logro de los aprendizajes logrados por las estudiantes puede entenderse por la naturaleza de la forma del trabajo con comunidad; bajo una modalidad tradicional de prácticas pedagógicas los procesos de aprendizaje son planificados prioritaria o exclusivamente en relación con la adquisición de conocimientos teóricos, con didácticas centradas en las conferencias magistrales, la lectura de textos y formas acotadas y no contextualizadas de experimentación. Los programas de estudio enfatizan la adquisición de conocimientos disciplinarios más que la integración interdisciplinaria de saberes y el desarrollo de las habilidades o competencias necesarias para el ejercicio de la profesión. La evaluación no prioriza la relevancia social de las actividades docentes y de investigación (CLAYSS, 2014).

2) Bajo un modelo de trabajo como el que ofrece la modalidad A+S, las prácticas pedagógicas se enriquecen en cuanto a que las experiencias ofrecen alta calidad de servicio solidario y un alto grado de integración con los aprendizajes formales. Se identifican como aprendizaje-servicio cuando la misma actividad tiene de manera simultánea objetivos de compromiso social y de aprendizaje evaluables, y ofrece a los estudiantes oportunidades de poner en juego no sólo valores y actitudes solidarias, sino también conocimientos y competencias específicas vinculadas a su perfil profesional. Los destinatarios de la práctica son la población atendida y los estudiantes, ya que ambos se benefician con el proyecto. El foco está puesto en la adquisición de aprendizajes y en el mejoramiento de las condiciones de vida de una comunidad concreta.

3) Por último, para la tercera categoría (aportes de la experiencia A+S a la formación profesional), se puede indicar que el aporte que brinda la experiencia de formación es importante en el desarrollo profesional y personal del estudiante, pues se planifica, ejecuta y evalúa un servicio solidario destinado a atender necesidades reales y sentidas de una comunidad, protagonizado activamente por los estudiantes desde el planeamiento a la evaluación, y articulado de acuerdo 
con los contenidos de aprendizaje (contenidos curriculares o formativos, reflexión, desarrollo de competencias para la ciudadanía y el trabajo, y la investigación) (Tapia, 2009, p. 21). En el aprendizaje-servicio el conocimiento se utiliza para mejorar algo de la comunidad y el servicio se convierte en una experiencia que proporciona conocimientos y valores. Aprendizaje y servicio quedan vinculados por una relación circular en la que ambas partes salen beneficiadas: el aprendizaje adquiere sentido cívico y el servicio se convierte en un taller de valores y saberes (Puig, 2009, p. 9).

En resumen, en cuanto a los criterios de eficacia planteados para el A+S se alcanzan aspectos como la intensidad de la actividad, significativa para el logro de aprendizajes profundos e integrales, permitiendo el protagonismo juvenil.

- El diagnóstico de necesidades realizado permite el protagonismo a la comunidad y la inserción efectiva de ellos en el proyecto.

- El diseño considera los intereses de los Estudiantes, incluye el conocimiento sociocultural previo de la realidad de la comunidad y la planificación prevista logra cumplirse.

- El proyecto en sí permite una alianza con la comunidad, constitución de redes estratégicas para el logro de la misión institucional, estipula momentos planificados de reflexión en el cual participan todos los actores involucrados.

- Se considera en la evaluación el impacto personal de la actividad en el estudiante.

- La actividad permite la efectiva satisfacción en la comunidad incluyendo sus intereses y perspectivas, pues los actores comunitarios son parte activa de la actividad.

No obstante dichos logros, se hace necesario considerar elementos que se levantan como nudos críticos en este estudio: 1) Potenciar los procesos de formación e inducción en el tema a docentes, así como monitoreo y acompañamiento del equipo de FHC; 2) Revisión del "ambicioso" itinerario de Servicio propuesto en el diseño por la Carrera (acotar a una actividad en el itinerario y especificar competencias genéricas); y 3) Este tipo de actividades requiere la instalación de prácticas pedagógicas fuera del límite urbano, lo que implica contemplar recursos de traslado para las sesiones de trabajo en campo y velar por las condiciones de conectividad territorial.

\section{Conclusiones}

De la experiencia de trabajo analizada para este artículo, se puede señalar que los resultados no difieren exponencialmente de otras experiencias de formación en modalidad A+S, ya que permiten sustentar este tipo de metodologías para el crecimiento personal y profesional de estudiantes de pregrado. En cuanto al primer aspecto, estas metodologías son expresiones de un nuevo paradigma -que podría parecer lejano y utópico, pero que ya está en práctica en muchas partes del mundo y que enriquecen y engrandecen al ser humano y moviliza la capacidad de entender lo que somos como sociedad (Butin, 2005; Tapia, 2006).

En el desarrollo profesional permiten a los jóvenes aplicar lo aprendido al servicio de sus comunidades, desarrollar habilidades para la vida, el trabajo y la participación ciudadana permite contribuir con el desarrollo local y mejorar la educación. La investigación muestra que el A+S fomenta la adquisición de conocimientos, habilidades, actitudes y valores en estudiantes de educación superior (Jouannet et al., 2013); los resultados permiten señalar de acuerdo que A+S fue una potente herramienta para trabajar con y por la comunidad, pues fomenta la cohesión social y la convivencia intercultural, y las conecta con la Educación Formal.

Frente a este escenario la docencia también enfrenta nuevos desafíos, pues bajo la perspectiva del A+S no sólo es necesario contar con docentes expertos en sus materias, sino con profesores que sepan cómo hacer que sus alumnos dominen estos conocimientos y encuentren las formas apropiadas para que las 
pongan en práctica de manera responsable, considerando la realidad social actual (Jouannet et al., 2013)

Finalmente, experiencias como éstas evidencian que las experiencias de A+S aportan y fortalecen futuras líneas de investigación, y logran articular las misiones de docencia, investigación y extensión. En las universidades, los procesos de articulación entre Docencia, Investigación y Extensión se llevan a cabo con diversos grados de intencionalidad y eficacia, pero hay signos esperanzadores frente a la creciente tendencia para establecer puentes entre pensamiento y acción, entre rigurosidad académica y compromiso social (CLAYSS, 2014).

\section{Agradecimientos:}

Agradecemos a la Cátedra Fray Bartolomé de las Casas por su apoyo en la realización de esta investigación, instancia que financió la presentación de esta experiencia en el Congreso APSU7-Santiago de Compostela (octubre, 2016).

\section{Referencias}

Asociación Nacional de Facultades y Escuelas de Contaduría y Administración. (junio, 2013). Evaluación del aprendizaje y vinculación universitaria. Trabajo presentado en la LIV Asamblea Nacional ANFECA. Jalisco, México.

Ávalos, B. (2014). La formación inicial docente en Chile: tensiones entre políticas de apoyo y control. Estudios pedagógicos, 40(especial).

Ávalos, B. y Matus, C. (2010). La formación inicial docente en Chile desde una óptica internacional. Informe nacional del estudio internacional IEA TEDS-M. Santiago: Ministerio de Educación.

Barber, M., \& Mourshed, M. (2007). How the world's best performing systems come out on top. Londres: McKinsey \& Co.

Bennett, M. J. (1986). A developmental approach to training for intercultural sensitivity. International Journal of Intercultural Relations, 10(2), 179-196.

Bennett, M. J. (1993). Towards ethnorelativism. A developmental model of intercultural sensitivity. En R. M. Paige (Ed.), Education for the Intercultural Experience (pp. 21-71). Yarmouth, ME: Intercultural Press.

Bennett, M. J. (2004). Becoming interculturally competent. En J. Wurzel (Ed.), Toward multiculturalism: a reader in multicultural education (pp. 62-77). Newton, MA: Intercultural Resource Corporation.

Butin, D. W. (Ed). (2005). Service-learning in higher education: critical issues and directions. Nueva York, Palgrave Macmillan.

Centro Latinoamericano de Aprendizaje y Servicio Solidario. (2014). La propuesta pedagógica del aprendizaje-servicio solidario en la Universidad. Texto complementario para los participantes en el programa latinoamericano de apoyo a universidades solidarias. Buenos Aires, Argentina.

Cooper, S. B., Cripps, J. H. y Reisman, J. I. (2013). Service-learning in deaf studies: impact on the development of altruistic behaviors and social justice concern. American Annals of the Deaf, 157(5), pp. 413-27.

Cox, C., Meckes, L. y Bascopé, M. (2010). La institucionalidad formadora de profesores en Chile en la década del 2000: Velocidad del mercado y parsimonia de las políticas. Pensamiento Educativo, Vols. 46$47,205-245$ 
Delors, J. (Comp). (1994). Los cuatro pilares de la educación, en La Educación encierra un tesoro (pp. 91103). (Informe a la UNESCO de la Comisión Internacional sobre la Educación para el siglo XXI). México:UNESCO.

Eyler, J. (2009). The power of experiential education. Liberal Education, 95(4), 24-31.

Flick, U. (2004). Introducción a la investigación cualitativa. España: Morata.

Furco, A. y Billig, S. (Eds.). (2002). Service learning: the essence of pedagogy. Connecticut: Information Age Publishing.

Glaser, B. G. y Strauss, A. L. (1967). The discovery of Grounded Theory. Chicago: Aldine.

Groh C. J., Stallwood L. G. y Daniels, J. J. (2011). Service-learning in nursing education: its impact on leadership and social justice. Nursing Education Perspectives, 32(6), 400-405.

Jenkins, A. y Sheehey, P. (2011). A checklist for implementing service-learning in higher education. Journal of Community Engagement and Scholarship, 4(2), 52-60.

Jouannet, M., Salas, M. y Contreras (2013). Modelo de implementación de aprendizaje servicio (A+S) en la Uc. Una experiencia que impacta positivamente en la formación profesional integral. Calidad en la educación, 39.

Krippendorff, K. (1990). Metodología de análisis de contenido: teoría y práctica. Barcelona: Paidós.

Mason, F. (2015). De experiencia intercultural a experiencia transcultural. Una nueva mirada a los programas de intercambio internacional estudiantil dentro del marco de una educación. Tesis de Magister, Universidad Alberto Hurtado, Santiago de Chile.

Ministerio de Educación, Ciencia y Tecnología. (2006). Experiencias ganadoras del premio presidencial "Prácticas Solidarias en Educación Superior" 2004. Argentina: Autor.

Ministerio de Educación. (2012). Estándares de formación para las carreras de educación parvularia. Santiago de Chile: Autor.

Molina, F. (2002). Sociología de la educación intercultural: investigación y docencia en el Espacio Europeo de Educación Superior. Recuperado de https://www.researchgate.net/publication/28179933 Sociologia de la educacion intercultural investig acion y docencia en el Espacio Europeo de Educacion Superior

OCDE. (2005). Teachers matter: attracting, developing and retaining effective teachers. Ediciones OCDE.

Pedraja, L. y Rodríguez-Ponce, E. (2012). Los estilos de liderazgo y la gestión de recursos humanos: una reflexión acerca del impacto sobre el desempeño de los estudiantes en la educación media. Ingeniare, 20(3), 376-385.

Puig, J. M. (Coord.) (2009). Aprendizaje servicio (ApS). Educación y compromiso cívico (Col. Crítica y fundamentos, 26). Barcelona: Graó.

Quivy, R. y Campenhoudt, L. (1998). Manual de investigación en ciencias sociales. México: Limusa.

Ruiz, J. I. (1996). Metodología de la investigación cualitativa. Bilbao: Universidad de Deusto.

Sagastizabal, M. A. (Coord.). (2009). Aprender y enseñar en contextos complejos multiculturalidad, diversidad y fragmentación. Buenos Aires: Noveduc. 
Sorrells, K. y Nakagawa, G. (2008). Intercultural communication praxis and the struggle for social responsibility and social justice. En O. Swartz (Ed.), Transformative communication studies. Culture, hierarchy, and the human condition (pp. 17-43). Lancaster: Troubador Publishing.

Strauss, A. y Corbin, J. (2002). Bases de la investigación cualitativa. Técnicas y procedimiento para desarrollar la Teoría Fundamentada. Colombia: Universidad de Antioquia.

Tapia, M. N. (2006) La solidaridad como pedagogía. Buenos Aires: Ciudad Nueva.

Tapia, M. N. (septiembre, 2009). Aprendizaje-servicio y calidad educativa. Actas del 110. Seminario Internacional "Aprendizaje y Servicio Solidario". Argentina. Recuperado de www.me.gov.ar/edusol

Trinidad, A., Carrero, V. y Soriano, R. (2006). Teoría fundamentada. La construcción de la teoría a través del análisis interpretacional. Madrid: Centro de Investigaciones Sociológicas.

UNESCO. (2006). Directrices de la UNESCO sobre la educación intercultural. París: Autor. Recuperado de http://unesdoc.unesco.org/images/0014/001478/147878s.pdf

Universia. (2010). II Encuentro de Rectores Universia, "Por un Espacio Iberoamericano del Conocimiento Socialmente Responsable". Guadalajara, México. 